\title{
Gold nanoparticle integrated with nanostructured carbon and quantum dots: synthesis and optical properties
}

\author{
Yuan $\mathrm{Li}^{1} \cdot$ Nitin Chopra ${ }^{1,2,3}$
}

Published online: 22 July 2015

(C) The Author(s) 2015. This article is published with open access at SpringerLink.com

\begin{abstract}
Multilayer graphene shell encapsulated gold nanoparticle - quantum dot hybrids were derived by combining wetchemical, thermal, and covalent chemistry approaches. Uniformly patterned gold nanoparticles on a silicon substrate were obtained via gold film deposition in an electroless method followed by a thermal dewetting process. The resulting gold nanoparticles were further surface oxidized and utilized as catalysts for the chemical vapor deposition growth of multilayer graphene shell encapsulated on the gold nanoparticles (referred as "multilayer graphene shell encapsulated Au nanoparticle" or graphene nanoparticles (GNPs)). As a next step, the surface of GNPs was modified to result in carboxylic $(-\mathrm{COOH})$ functionalities, which enabled carbodiimide-based covalent linking of amine-terminated $\mathrm{CdS}_{x} \mathrm{Se}_{1-x} @ \mathrm{ZnS}$ quantum dots (QDs) on the GNP surface. The GNPs and GNP-QD heterostructures were characterized using scanning and transmission electron microscopy for size, morphology, spatial distribution, and crystal structure evaluation. In addition, UV-vis, fluorescence spectroscopy, and discrete dipole approximation (DDA) modeling were utilized for understanding the band gap energies, fluorescence quenching, and light-matter interactions of the derived hybrids/heterostructures.
\end{abstract}

Electronic supplementary material The online version of this article (doi:10.1007/s13404-015-0163-3) contains supplementary material, which is available to authorized users.

Nitin Chopra

nchop2@gmail.com

1 Department of Metallurgical and Materials Engineering, The University of Alabama, Box 870202, Tuscaloosa, AL 35401, USA

2 Department of Biological Sciences, The University of Alabama, Box 870344, Tuscaloosa, AL 35401, USA

3 Department of Chemistry, The University of Alabama, Box 870336, Tuscaloosa, AL 35401, USA
Keywords Graphene · Gold nanoparticles · Quantum dots · Optical properties, discrete dipole approximation

\section{Introduction}

The surface modification of photonic nanostructures such as gold $(\mathrm{Au})$ nanoparticles can lead to novel physical phenomena including selective light-matter interactions and rapid energy transfer processes [1-3]. The surface modification of $\mathrm{Au}$ nanoparticles could be achieved by using ligand coatings, inorganic material shells, or covalent/physical adsorption of nanoparticles or quantum dots of another material [4]. However, the challenge is to achieve stable coating around $\mathrm{Au}$ nanoparticles, which prevents the exposure of Au nanoparticles to unwanted solution environment, prevent their aggregation, and yet retain their properties [4-6]. Overcoming this challenge will also enable conservative utilization of Au nanoparticles in photonic applications. In addition, the need is for imparting diverse surface chemistry to Au nanoparticles. A promising approach is to encapsulate Au nanoparticles in a sub-5-nm-thin carbon or graphene shell. Such modification of Au nanoparticles can lead to hierarchical heterostructures by utilizing rich carbon chemistry. An attractive architecture will be to bind quantum dots [7-9] onto carbon encapsulated $\mathrm{Au}$ nanoparticles. The carbon component can also influence the light-matter interactions for such hybrid systems, for example, by reducing scattering effects [10]. Such hybrid systems can be envisioned as a new kind of metamaterial architectures.

Recently, surface decoration of Au nanoparticles with chemically exfoliated graphene was reported for biosensors and bioelectronics [11-13]. However, the corrosive nature of the exfoliation process and the inability of wrapped graphene to form an impervious shell around Au nanoparticles limited their electrical and optical properties. In this regard, encapsulation of $\mathrm{Au}$ 
nanoparticles in a sub-5-nm-thick carbon shell or a multilayer graphene shell has been recently demonstrated by the authors using an in situ carbon growth approach [14-16]. This scalable approach employs a chemical vapor deposition (CVD) of multilayer graphene shells directly onto patterned $\mathrm{Au}$ nanoparticles (referred as "multilayer graphene shell encapsulated Au nanoparticle" or graphene nanoparticles (GNPs)) [10-12]. The presence of multilayer graphene shell (sub-5 nm thickness) influenced the optical properties and stability (chemical and thermal) of the encapsulated Au nanoparticles [10-16]. In addition, carbon surface chemistry allows for target-receptor bioanalysis and sensing [16].

In this short note, we report an approach for the controlled patterning of multilayer graphene-modified Au nanoparticles (or GNPs) on a silicon substrate and covalent linking of GNPs with semiconducting $\mathrm{CdS}_{x} \mathrm{Se}_{1-x} @ \mathrm{ZnS}$ quantum dots (referred as GNP-QD heterostructures). High-resolution electron microscopy was utilized to study the crystal structure, interfaces, and binding of quantum dots with GNPs. Optical properties of heterostructures were analyzed using UV-vis and fluorescence spectroscopy. For understanding the light-matter interactions, discrete dipole approximation (DDA) modeling method was utilized to simulate surface plasmonic characteristics of GNPs and GNP-QD heterostructures.

\section{Experimental section}

\section{Materials and methods}

Silicon (Si) wafers (111) were purchased from IWS (Colfax, CA). Potassium gold (III) chloride was purchased from SigmaAldrich (St. Louis, MO). Hydrogen fluoride $(\sim 50 \mathrm{wt} \%)$ was purchased from VWR (Atlanta, GA). Ammonium fluoride was purchased from Alfa Aesar (Ward Hill, MA). 1-Ethyl3-(3-dimethylaminopropyl) carbodiimide (EDC) and Nhydroxysuccinimide (NHS), 2-(N-morpholino) ethanesulfonic acid (MES) were purchased from Thermo Scientific (Rockford, IL). Amine-terminated $\mathrm{CdSe}_{x} \mathrm{~S}_{1-x} / \mathrm{ZnS}$ core/shell fluorescent quantum dots were purchased from Cytodiagnostics (Burlington, Canada). Deionized (DI) water (18.1 M $\Omega-\mathrm{cm})$ was obtained using a Barnstead International DI water system (E-pure D4641). All chemicals were used without further purification. Buffered oxide etch (BOE) solution was prepared by mixing $\sim 40 \mathrm{wt} \% \mathrm{NH}_{4} \mathrm{~F}$ and $\sim 50 \mathrm{wt} \% \mathrm{HF}$ in DI water (volume ratio of $6: 1)$. The GNP growth was carried out in a Lindberg blue threezone tube furnace (Watertown, WI) with a quartz tube purchased from ChemGlass (Vineland, NJ).

\section{Synthesis of multilayer graphene shell encapsulated Au nanoparticles}

Au film was deposited on the silicon substrate using acid-based wet-chemical method $[17,18]$. In this method, cleaned silicon substrate was treated with $\mathrm{BOE}$ solution to remove the surface oxide layer. This was followed by immersing the substrate in the acidic solution comprised of $\sim 1 \mathrm{mM} \mathrm{KAuCl}{ }_{4}$ in $\sim 1 \mathrm{wt} \%$ HF. This reaction (also referred as electroless or galvanic deposition) was carried out for 45, 90, and $120 \mathrm{~s}$ to result in Au films with different thicknesses. The substrate was washed with DI water, dried, and subsequently annealed at $850{ }^{\circ} \mathrm{C}$ in air for $10 \mathrm{~min}$. This annealing step led to de-wetting of $\mathrm{Au}$ film and resulted in Au nanoparticles patterned onto the silicon substrate. Growth of multilayer graphene shell around Au nanoparticles (GNPs) was accomplished in a CVD process [14-16] for patterned Au nanoparticles after dewetting of three samples (i.e., Au films) on silicon substrates deposited for $45 \mathrm{~s}$ (GNP_45), $90 \mathrm{~s}$ (GNP_90), and $120 \mathrm{~s}$ (GNP_120). The GNP growth was conducted as described next. Briefly, Au nanoparticles patterned on silicon substrate were treated with oxygen plasma for $15 \mathrm{~min}$ to result in surface gold oxide. This was followed by the CVD growth of multilayer graphene shell at $\sim 675^{\circ} \mathrm{C}$ for $1 \mathrm{~h}$. Xylene was used as the carbon source. The carrier gas was $10 \% \mathrm{H}_{2} /$ Ar with a total flow rate of $1.15 \mathrm{SLM}$. This process resulted in patterned growth of GNPs on the silicon substrate. Finally, the - $\mathrm{COOH}$ derivatization on the GNPs was carried out by treating the as-grown GNPs with oxygen plasma for $15 \mathrm{~s}$ (power $120 \mathrm{~W}$, oxygen pressure $300 \mathrm{mTorr}$ ).

\section{Covalent binding of quantum dots on GNPs}

Commercially bought and amine $\left(-\mathrm{NH}_{2}\right)$-terminated $\mathrm{CdS}_{x} \mathrm{Se}_{1-}$ ${ }_{x} @ \mathrm{ZnS}$ quantum dots were covalently linked with $(-\mathrm{COOH})-$ derivatized GNPs via the carbodiimide chemistry [19-21]. Briefly, the (-COOH)-terminated GNPs patterned on the silicon substrate were immersed in a $\sim 50 \mathrm{mM}$ MES buffer $(\mathrm{pH}=6.1)$ solution containing $\sim 2 \mathrm{mM}$ EDC and incubated for 10 min under gentle shaking. This was followed by addition of $\sim 4 \mathrm{mM} \mathrm{NHS}$ and further incubation for $1 \mathrm{~h}$. This process allowed the formation of stable amine-reactive NHS ester on the surface of GNPs. The substrate was gently washed with PBS buffer $(\mathrm{pH}=7.4)$ and then immersed in the PBS buffer $(\mathrm{pH}=7.4)$ containing $2 \mathrm{mM}$ $\left(-\mathrm{NH}_{2}\right)$-terminated $\mathrm{CdSe}_{x} \mathrm{~S}_{1-x} / \mathrm{ZnS}$ quantum dots. This reaction was continued for $12 \mathrm{~h}$ to form the amide bonds (-CONH-) and result in the covalently linked GNP-QD heterostructures. The final product was washed with DI water and dried in $\mathrm{N}_{2}$. For the measurement of fluorescence spectra, this carbodiimide chemistry was conducted in solution. The GNPs of each size were collected by sonicating a substrate of $2 \times 2 \mathrm{~cm}^{2}$ in DI water for $30 \mathrm{~min}$. For binding to QDs, similar carbodiimide chemistry process was conducted as described above. In all the samples, the starting QD concentration was kept constant.

\section{Characterization methods}

FE-SEM (JEOL-7000) and Tecnai F-20 TEM were used to characterize the morphology, crystal structure, and/or 
interfaces of the GNPs and GNP-QD heterostructures. The dimension, number density, and shell thickness were estimated accordingly. UV-vis absorbance spectra were obtained using DH-2000 UV-VIS-NIR light source. Fluorescence spectroscopy (JASCO FP-8200) was used to characterize the fluorescence quenching effect of GNP-QD heterostructures.

The band gap energies for the as-purchased quantum dots and the GNP-QD heterostructures were further determined using Eq. (1) [22, 23]:

$a h v=A\left(h v-E_{g}\right)^{n}$

where $a$ is absorbance coefficient, $A$ is a constant, $h v$ is the energy of light and $n$ is a constant depending on the nature of the electron transition ( $n$ assumes 1/2,2,3/2, and 3 for allowed direct, allowed indirect, forbidden direct, and forbidden indirect transitions, respectively). $\mathrm{CdS}_{x} \mathrm{Se}_{1-x} / \mathrm{ZnS}$ quantum dots have been reported as an allowed direct semiconductor; as a result, the optical band gaps were calculated by extrapolating straight lines of the Tauc plots $\left[(a h v)^{2}\right.$ vs. $\left.h v\right]$ to the intercept where $(a h v)^{2}=0[24,25]$.

Discrete dipole approximation (DDA) method was used to simulate the plasmonic properties of the GNPs and GNP-QD heterostructures [26]. This method is based on the solution of 3D Maxwell equation using the DDSCAT code developed by Draine and Flatau [27, 28]. Briefly, DDSCAT 7.2 was utilized to estimate the normalized electric field or surface plasmon generation for nanostructure/heterostructure targets. The normalized electric field intensity is defined as the strength ratio between the electric field generated near the nanostructures and the incident electric field $\left(|\mathrm{E}| /\left|\mathrm{E}_{0}\right|\right)$. The geometrical targets were created using the 3D max software for GNPs and GNPQD heterostructures with various sizes. The effective radius of the targets was calculated via: $r_{\mathrm{eff}}=(3 V / 4 \pi)^{1 / 3}$, where $V$ is the total volume of the 3D targets and is calculated with $V=N d^{3}$ ( $N$ is the number of dipoles and $d$ is the lattice spacing in cubic array). Near-field electric field distributions were extracted using the DDFIELD utility in DDSCAT 7.2 and processed using MayaVi 2 from Python $(x, y)$.

\section{Results and discussion}

The patterning of $\mathrm{Au}$ nanoparticles on the silicon substrate was achieved in an electroless or galvanic deposition process $[17,18]$ followed by the high-temperature annealing. Electroless $\mathrm{Au}$ deposition reaction was performed for different durations $(45,90$, and $120 \mathrm{~s})$. Increasing reaction time led to thicker $\mathrm{Au}$ films. The electroless deposition process involved the replacement reaction between $\mathrm{Au}^{3+}$ and $\mathrm{Si}$ in an aqueous solution containing $\mathrm{F}^{-}$, which resulted in deposition of a uniform $\mathrm{Au}$ film on the silicon substrate [29]. Followed by this, the dewetting of $\mathrm{Au}$ films resulted in uniformly sized and patterned Au nanoparticles on the silicon substrate. For the growth of multilayer graphene shell, Au nanoparticles were surface oxidized [14] and utilized as catalysts in a xylenebased CVD process $[14,15]$.

Figure $1 \mathrm{a}-\mathrm{c}$ shows the SEM images of the GNPs (GNP_45, GNP_90, and GNP_120) obtained for three different samples with increasing Au nanoparticle sizes. The microscopic images clearly show that GNPs were uniformly patterned onto the substrate (Fig. 1a-c), and a multilayer graphene shell (Fig. 1d-f) was observed with a core Au nanoparticle encapsulated within. The carbon inter-planar spacing is observed to be between 0.37 and $0.40 \mathrm{~nm}$ (Fig. 1d-f). This carbon inter-planar spacing larger than that for $c$-axis lattice spacing of graphite $(\sim 0.34 \mathrm{~nm})$ could be attributed to the curvature state of graphene shell [15]. Using microscopic characterization, the GNP size, inter-particle spacing, and spatial density for various samples were estimated (Table 1). It can be noted (Fig. 1g) that the GNP size increases with increasing electroless deposition duration of Au film. This suggests that longer deposition time led to thicker Au film, which further resulted in larger-sized Au nanoparticles for a fixed dewetting condition. In addition, the size distribution of GNPs was broader for larger-sized GNPs than that for smaller-sized ones. This indicates that Ostwald's ripening effect and surface migration of species enhanced with the size of the nanoparticles [15]. It is also noted that with the increase of GNP size, an increasing trend in inter-particle spacing and decreasing trend in spatial density of GNPs is observed. The thickness of multilayer graphene shell corresponding to Fig. 1a-c is observed to be $\sim 2.5 \pm 0.3, \sim 3.8 \pm 0.2$, and $\sim 4.2 \pm 0.5 \mathrm{~nm}$ for GNP_45, GNP_90, and GNP_120 samples, respectively. This increasing shell thickness with increasing GNP/Au nanoparticle size suggests that larger Au nanoparticles can result in thicker graphene shell.

The as-produced and patterned GNPs on the silicon substrate were subjected to plasma oxidation process to enable the formation of $-\mathrm{COOH}$ groups on the multilayer graphene shell. In addition, this controlled process also assists in removing the amorphous carbon present on the shell or etching the shell [30]. As a next step, these ( $-\mathrm{COOH})$-functionalized GNPs were covalently linked with $\left(-\mathrm{NH}_{2}\right)$-terminated $\mathrm{CdS}_{x} \mathrm{Se}_{1-x} / \mathrm{ZnS}$ quantum dots [19, 31]. Figure 2a-c shows TEM images of the resulting GNP-QD heterostructures that were derived using GNP 45, GNP 90, and GNP_120 samples. These three samples are referred as GNP-QD_1, GNP-QD_2, and GNP-QD_3. High-resolution TEM images (Fig. 2a, d, and e) clearly show the attached quantum dots with sizes between 4 and $5 \mathrm{~nm}$ and lattice spacing of $\sim 0.34 \mathrm{~nm}$ (which is corresponding to (111) plane of CdS). Since TEM images are 2D images, it is impossible to identify the spatial location of the quantum dots on GNPs, but their presence on the shell can be easily identified as shown in Fig. 2. As shown in Fig. 2a-c, it is clear that quantum dots are uniformly coated around the GNPs. Based 
Fig. 1 a-c SEM images of GNPs with different size that were uniformly patterned onto silicon substrate. $\mathbf{d}-\mathbf{f}$ TEM images of GNPs corresponding to $\mathbf{a}-\mathbf{c} . \mathbf{g}$ Histogram showing the distribution of GNP diameter for various GNP samples. $\mathbf{h}$ Histogram of GNP diameters and inter-particle spacing for various GNP samples
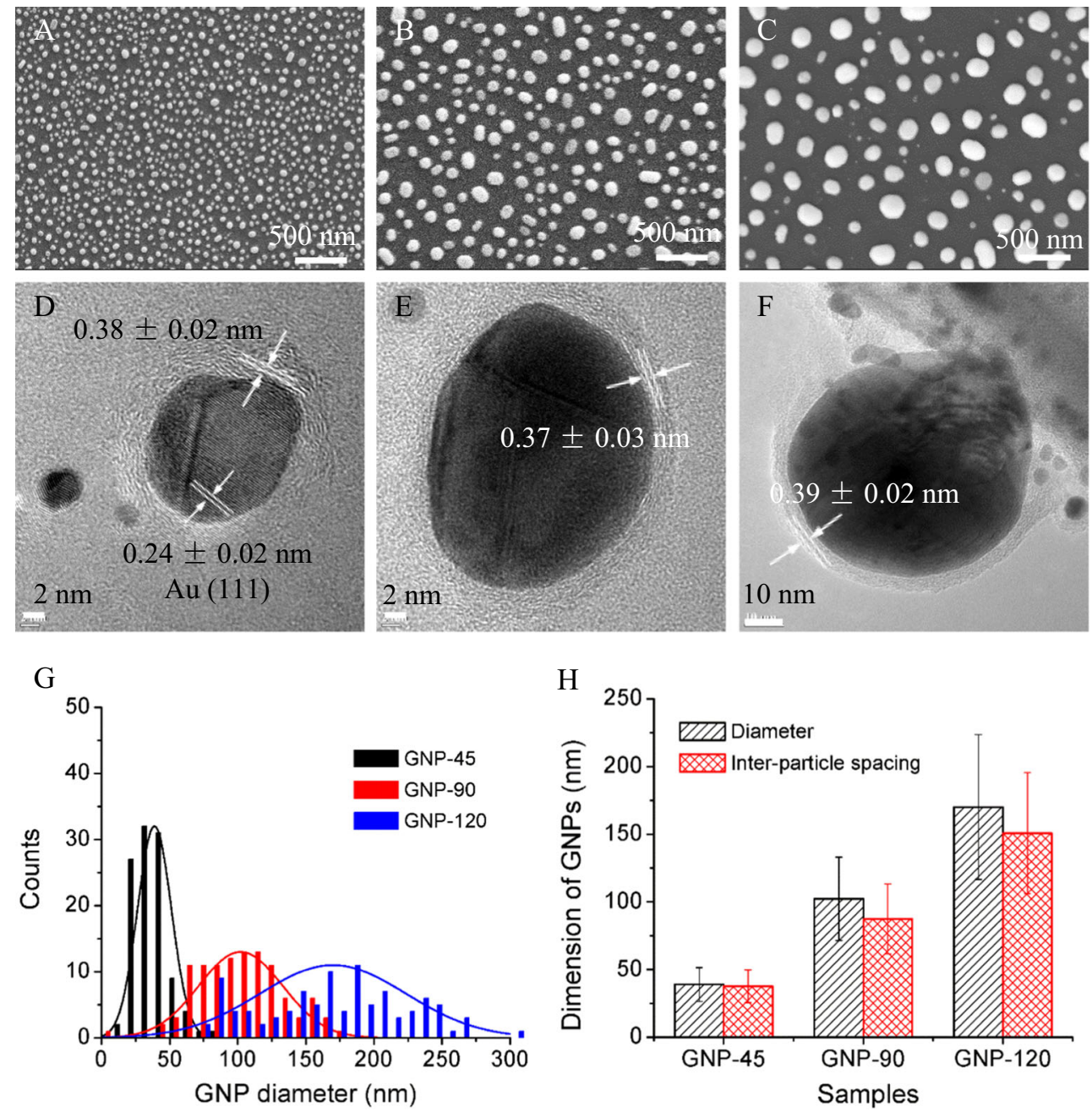

on this observation, the surface coverage of quantum dots on the GNPs is estimated to be $\sim 40 \%$ for GNP-QD_1, $18 \%$ for GNP-QD_2, and $15 \%$ for GNP-QD_3. The difference in QD surface coverage as a function of GNP size could be attributed to several factors such as GNP size including shell thickness and extent of carboxylic $(-\mathrm{COOH})$ derivatization. GNPs with smaller size have larger curvature of graphene shell, which induces larger strains on the surface as compared to that for the larger GNPs. Under the same plasma treatment conditions, the smaller GNPs with high surface energy are prone to result in more defects and the enhanced formation of $-\mathrm{COOH}$ functional groups $[30,32]$. As a results, due to the larger amount of -
$\mathrm{COOH}$ groups on smaller GNPs, the latter covalently attach to more number of $\mathrm{NH}_{2}$-terminated QDs. This unique approach presents a simple method to develop highly ordered assemblies comprised of multiple components (Au nanoparticles, multilayer graphene shell, and quantum dots).

It is known that Au nanoparticles and quantum dots have unique plasmonic and photonic characteristics [10, 33]. However, these characteristics are difficult to manipulate except by varying the size and aggregation of such photonic nanostructures. In this regard, mathematical modeling has predicted that GNPs can exhibit modulated plasmonic and photonic properties by simply varying the thickness of the multilayer

Table 1 Size, inter-particle spacing, and spatial density of various GNP samples with different size

\begin{tabular}{lccr}
\hline Samples & Diameter of GNPs $(\mathrm{nm})$ & Inter-particle spacing of GNPs $(\mathrm{nm})$ & Spatial density of GNPs $\left(10^{8} / \mathrm{cm}^{2}\right)$ \\
\hline GNP_45 & $38.87 \pm 12.47$ & $37.58 \pm 12.05$ & 104.56 \\
GNP_90 & $102.19 \pm 30.72$ & $87.42 \pm 25.86$ & 18.09 \\
GNP_120 & $169.92 \pm 53.59$ & $150.74 \pm 44.70$ & 8.55 \\
\hline
\end{tabular}

The sample name indicates a numerical value that corresponds to the duration of the initial electroless deposition of Au film 
Fig. 2 TEM images of the GNPQD heterostructures

corresponding to $\mathbf{a}$ GNP $45, \mathbf{b}, \mathbf{d}$ GNP 90, and c, e GNP 120 .

Note: $\mathbf{d}$ and e show the highresolution TEM for attached quantum dots on GNPs as shown in $\mathbf{b}$ and $\mathbf{c}$. The blue dotted lines indicate the regions observed at higher resolution
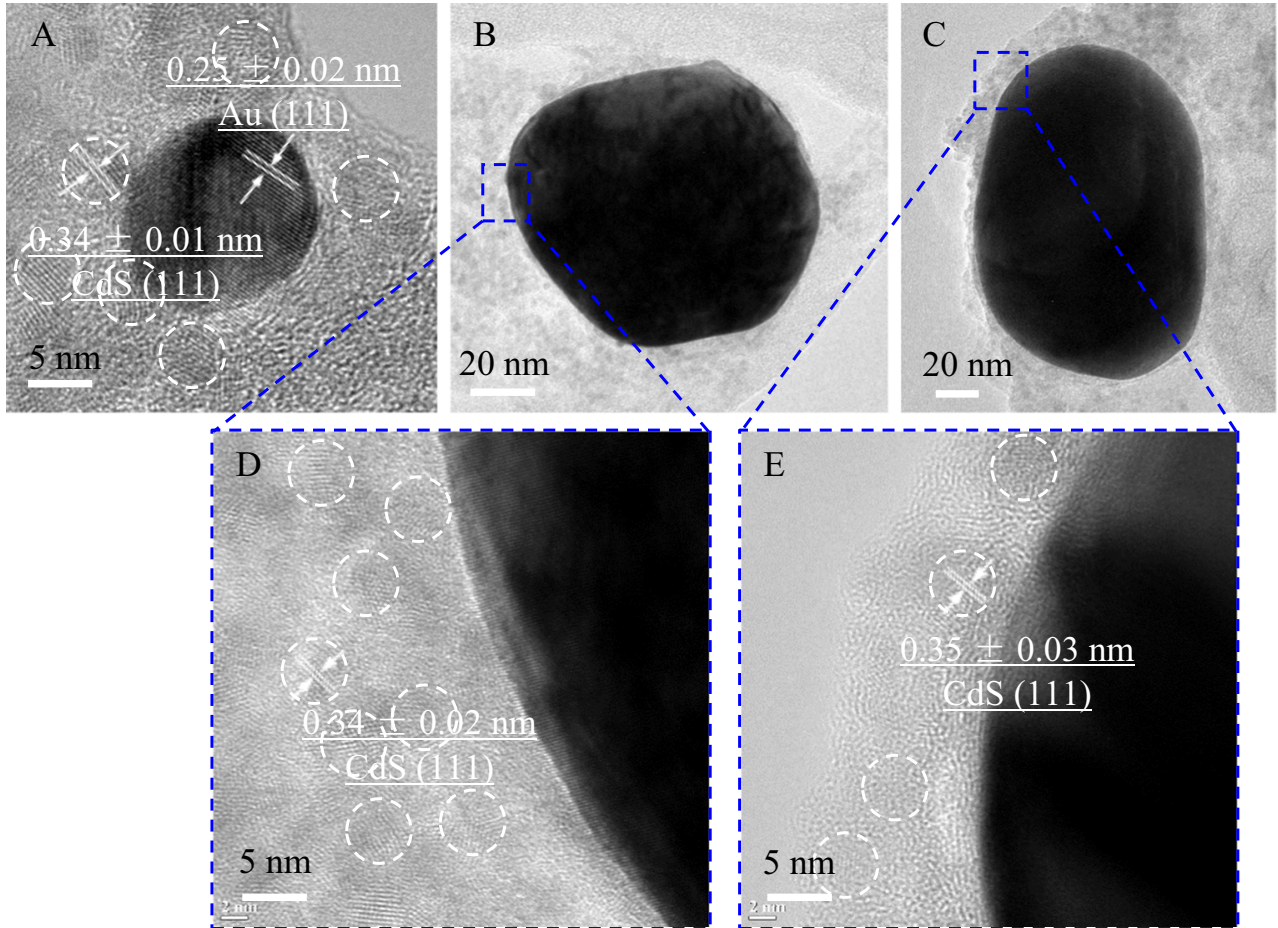

graphene shell [10]. In addition, multilayer graphene shell around Au nanoparticles allows for enhanced absorption with minimal scattering. Thus, GNP-QD heterostructures are of particular interest for optical applications. The optical properties of GNPs and GNP-QD heterostructures were studied using UV-vis absorbance spectroscopy (Fig. 3a). The asproduced GNPs showed broad absorbance peaks at $\sim 550$, $\sim 570$, and $\sim 590 \mathrm{~nm}$. These peaks could be attributed to the
Fig. 3 UV-vis absorbance spectra for a GNP samples and corresponding b GNP-QD heterostructures. c Tauc plots or $(\alpha h v)^{2}$ vs. $h v$ plots used for the band gap energies estimation of the as-purchased quantum dots and GNP-QD heterostructures. $\mathbf{d}$ Fluorescence spectra of the aspurchased quantum dots and GNP-QD heterostructures
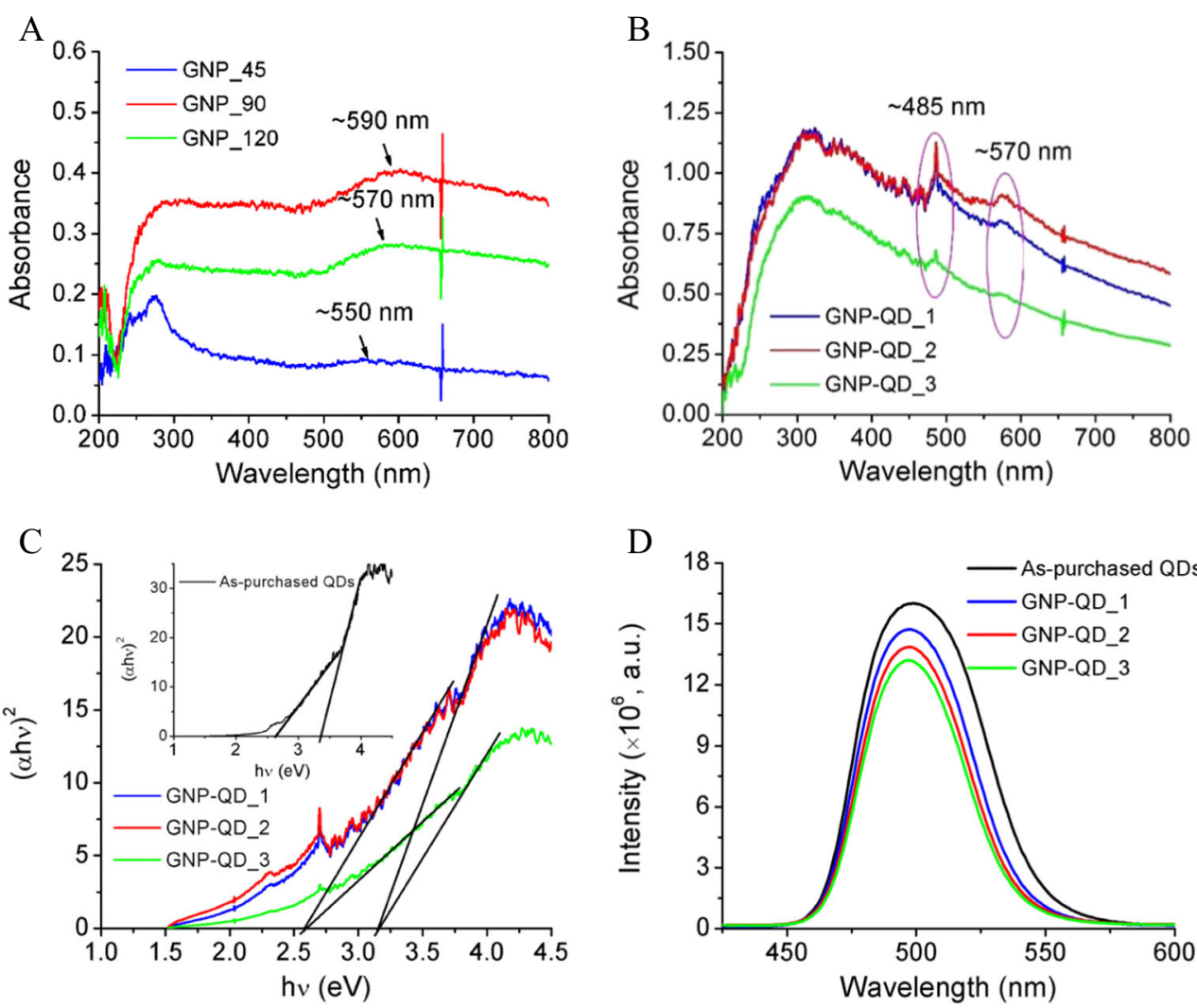
visible absorption of the encapsulated Au nanoparticle in GNP [34]. The red shift in this peak for the three GNP samples could be attributed to the different thickness of the multilayer graphene shell and the size of the encapsulated Au nanoparticle and is largest for GNP_90 sample [34]. Another absorbance peak was observed in the UV region $(280-290 \mathrm{~nm})$ and attributed to the overlapping of UV absorption of multilayer graphene shell and inter-band transition of Au nanoparticles [35, 36]. Suppression of this peak for thicker multilayer graphene shell was observed and could be due to the weakening of inter-band transition of Au nanoparticles or presence of amorphous carbon on thicker multilayer graphene shell. Apart from the peaks described above, all the three GNP-QD heterostructure samples exhibited peaks at $\sim 485 \mathrm{~nm}$ corresponding to the absorption of quantum dots (Fig. 3b). It must be noted that attachment of quantum dots onto GNPs resulted in red-shifted peaks (from $\sim 280-290 \mathrm{~nm}$ to $\sim 300-310 \mathrm{~nm}$ ) corresponding to $\mathrm{Au}$ inter-band transition or multilayer graphene shell. This could be attributed to light-matter interactions between GNP and quantum dots. Overall, these aspects indicate that individual component in hybrid assembly retained its optical behaviors and the presence of multilayer graphene shell modulated this further.

Tauc plots (Fig. 3c) were derived using the absorbance spectra in Fig. $3 b$ to evaluate the band gap energies of the GNP-QD heterostructures. The inset in Fig. 3c shows that
Fig. 4 3D targets for various (af) GNP samples and (g-k) GNPQD heterostructure samples. Note: GNP samples $(\mathbf{a}-\mathbf{c})$ were named as targets \#1-\#3 and the GNP-QD heterostructure samples (g-i) were named as targets \#4 \#6
A

B

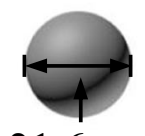

$21.6 \mathrm{~nm}$

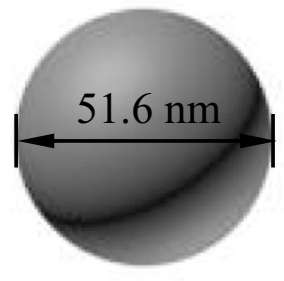

E
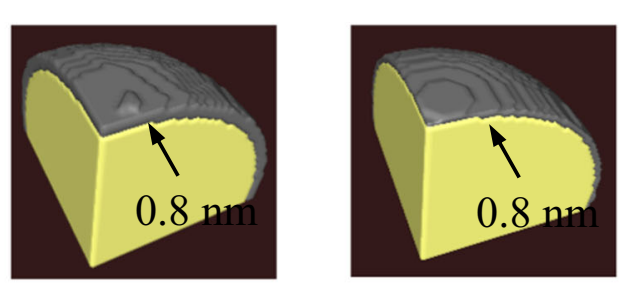

F

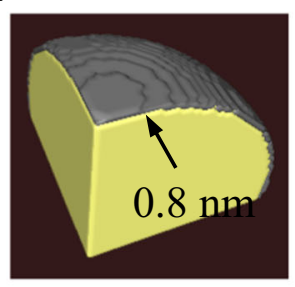

G
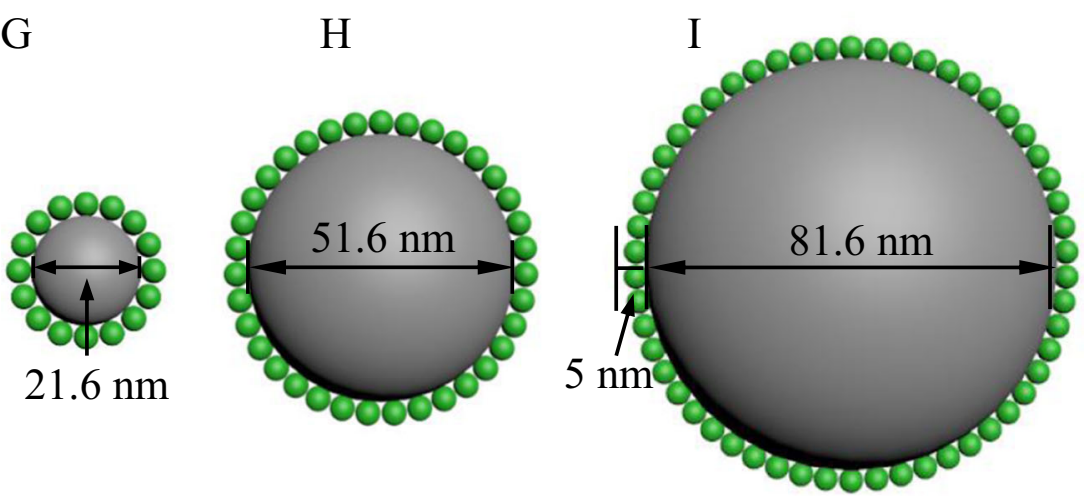

J

L

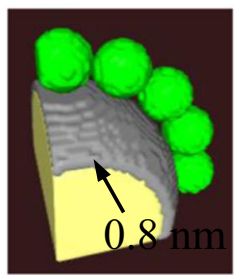

C

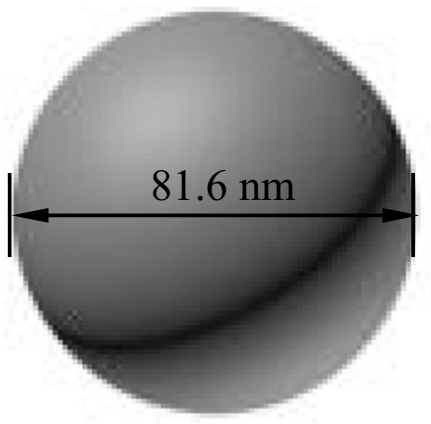

K
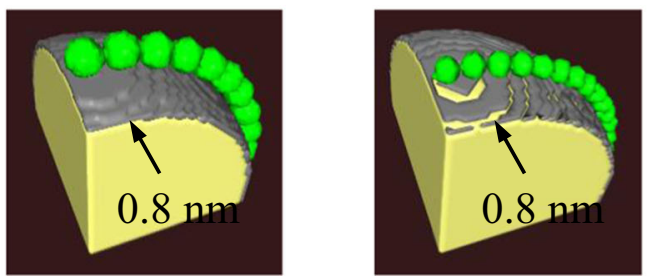
the as-purchased quantum dots exhibited two band gaps at $\sim 2.6$ and $\sim 3.4 \mathrm{eV}$, which are attributed to $\mathrm{CdS}$ and $\mathrm{ZnS}$, respectively [37]. One can observe in Fig. 3c that covalent linking of quantum dots onto GNPs has no significant effect on their band gap energies or band structure. This indicates that GNPs provide an excellent support for quantum dots while preserving the optical properties of latter in the heterostructure configuration. Such heterostructures with complex architectures hold strong potential for solar energy-harvesting devices, photocatalysis and Förster resonance energy transfer (FRET).

The fluorescence characteristics of GNP-QD heterostructures were characterized using the fluorescence spectroscopy. Since the emission of fluorescence from quantum dots occurs during the recombination of excited electron with the ground state, the intensity of fluorescence spectra is indicative of the charge recombination processes. The attachment of quantum dots onto electron-accepting surfaces ( $\mathrm{Au}$, graphene, or GNPs) prevents recombination of charge carriers. This resulted in fluorescence quenching effect (around $500 \mathrm{~nm}$, Fig. 3d) for the GNP-QD heterostructures, which show reduced fluorescence intensity [38]. In addition, the quenching effect becomes more pronounced with the increase of GNP size since larger gold nanoparticles have stronger surface plasma resonance, which strongly influences the electron transfer and separation in photovoltaic devices $[39,40]$. As compared with the as-purchased quantum dots, the quenching efficiency (estimated by the percentage ratio of spectral area in Fig. 3d) is 83.16, 75.93, and 70.43\% for GNPQD_1, GNP-QD_2, and GNP-QD_3, respectively.
GNP-QD heterostructures as derived in this study are potentially important for applications such as surface-enhanced Raman scattering (SERS), plasmonic-enhanced photocatalysis, $\mathrm{THz}$ devices, and optoelectronic devices. Aimed at these potential applications, it is necessary to understand the influence of multilayer graphene shell, encapsulated Au nanoparticles, and attached quantum dots on the plasmonic properties of GNP-QD heterostructures. This was achieved by simulating the extinction spectra (including absorbance and scattering) and surface electric field distribution for GNPs and GNP-QD heterostructures [27, 28]. The targets were comprised of an arrangement of dipoles for which extinction spectra and normalized electric field distributions were numerically solved [27]. Three spherical GNP targets (Fig. 4a-f) were derived by considering equally spaced $\mathrm{Au}$ nanoparticles (diameters $\sim 20$, $\sim 50$, and $\sim 80 \mathrm{~nm}$, referred as target $\# 1$, target $\# 2$, and target $\# 3$, respectively). The thickness multilayer graphene shell was $\sim 0.8 \mathrm{~nm}$. The GNP-QD targets were derived by considering quantum dots with diameter of $5 \mathrm{~nm}$ that were closely packed around three GNP targets (Fig. $4 \mathrm{~g}-\mathrm{k}$, referred as target \#4, target \#5, and target \#6). The simulation assumes air as an environment surrounding targets. The extinction efficiency is given by a combination of scattering and absorption as follows:

$Q_{\text {ext }}=Q_{\text {sca }}+Q_{\text {abs }}$

where $Q_{\text {ext }}$ is the extinction efficiency factor, $Q_{\text {sca }}$ is the scattering efficiency factor, and $Q_{\text {abs }}$ is the absorbance efficiency factor. The extinction spectra for all the GNPs and GNP-QD targets were deconvoluted into absorbance and scattering

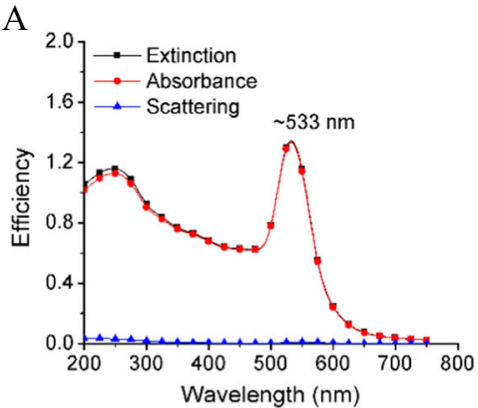

$\mathrm{D}$

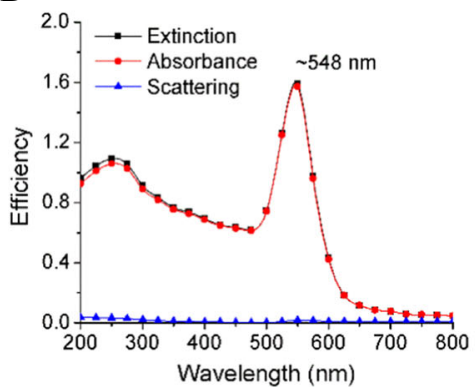

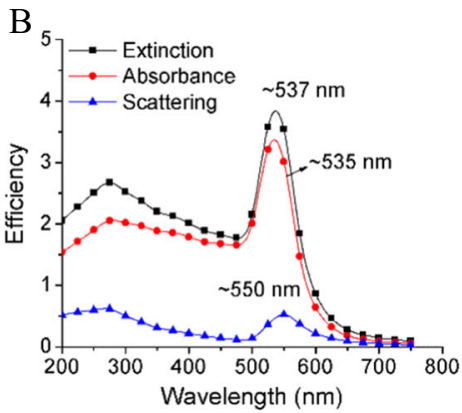

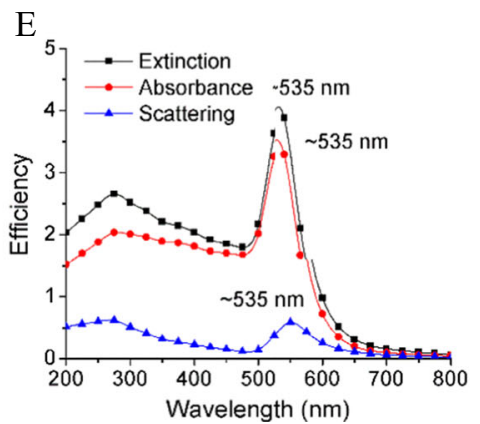

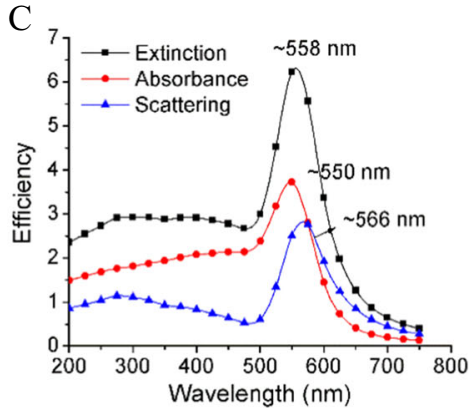

$\mathrm{F}$

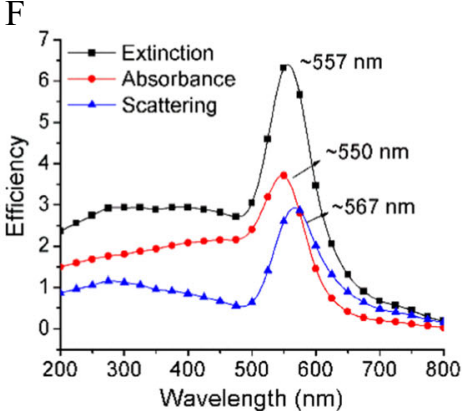

Fig. 5 Simulated extinction efficiency spectra as a function of wavelength for the GNP targets (a-c: targets \#1-\#3) and the GNP-QD heterostructure targets (d-f: targets \#4 \#6) shown in Fig. 4. Note: The extinction efficiency spectra were further deconvoluted into absorbance and scattering spectra for each target as shown as overlay in individual spectra plot 
spectra (Fig. 5, using Eq. 2). The main extinction peaks were observed between $\sim 530$ and $\sim 570 \mathrm{~nm}$ as well as at $\sim 270 \mathrm{~nm}$. These simulated peaks were consistent with experimental results (Fig. 3a, b) and attributed to the visible absorption of gold and the overlap of UV absorption of graphene and inter-band transition Au d-electrons [36, 41]. For small GNP and GNPQD targets $\left(R_{\mathrm{GNP}}=10 \mathrm{~nm}\right)$, the scattering component was significantly suppressed as compared to the absorption component (Fig. 5a, d) [35]. However, with increasing GNP size $\left(R_{\mathrm{GNP}}=\right.$ $25 \mathrm{~nm}$ and $40 \mathrm{~nm}$ ), the scattering for both, GNP and GNP-QD targets, were enhanced (Fig. 5b, c, e, and f). This observation can be well explained by the theoretical calculation reported earlier [35]. When nanoparticles are small compared to the wavelength of light, the scattering is in the Rayleigh regime. In that case, the scattering $\left(Q_{\text {sca }}\right)$ can be written as:

$Q_{\text {sca }}=\frac{8 \pi}{3} k^{4} R^{6} F(m)$
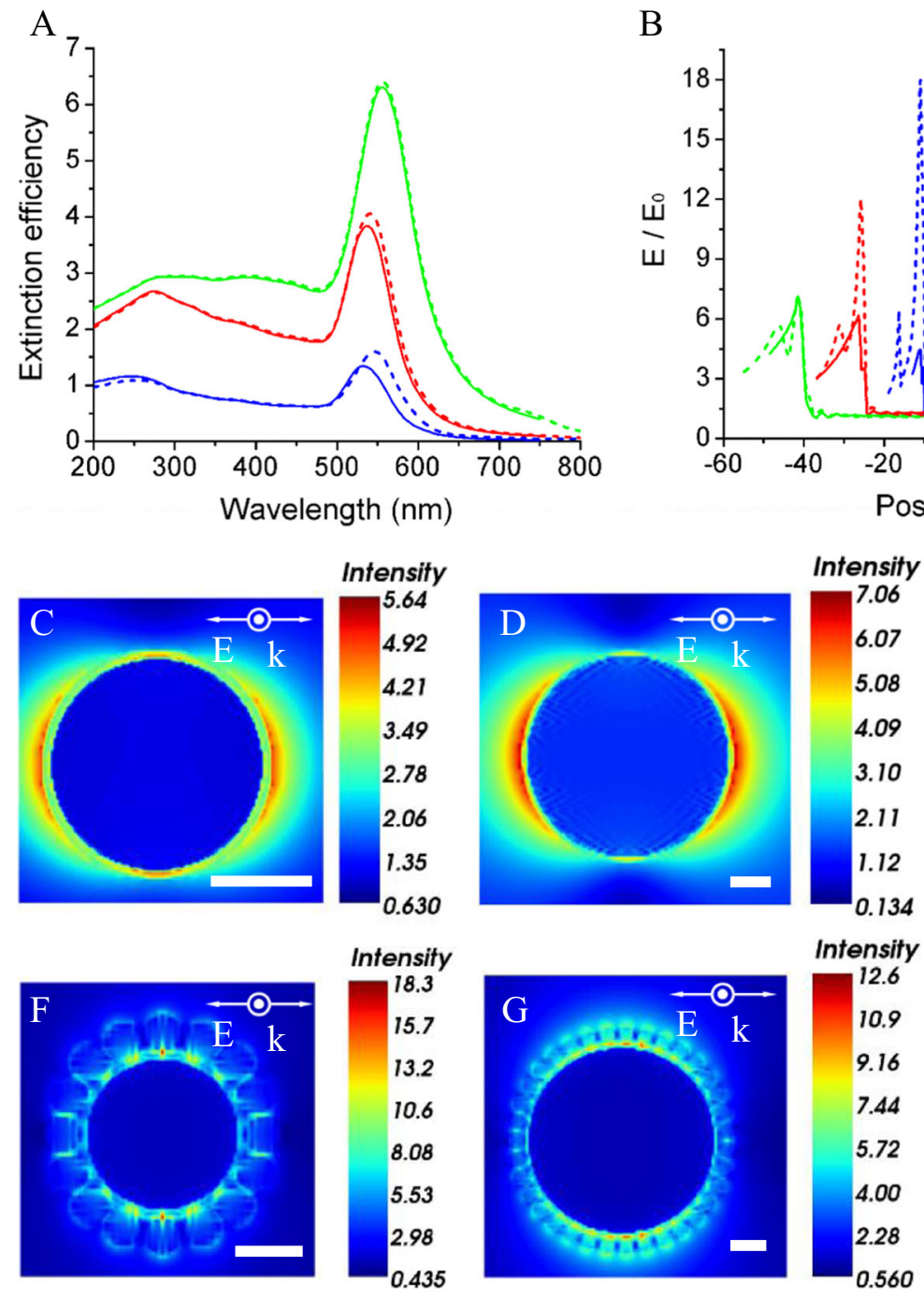

Fig. 6 a Simulated extinction efficiency spectra for the GNP and GNP$\mathrm{QD}$ heterostructure targets. b Normalized electric field intensity $\left(\mathrm{E} / \mathrm{E}_{0}\right) \mathrm{vs}$. positions (from center to the edge of target). $\mathbf{c}-\mathbf{h}$ Normalized electric field
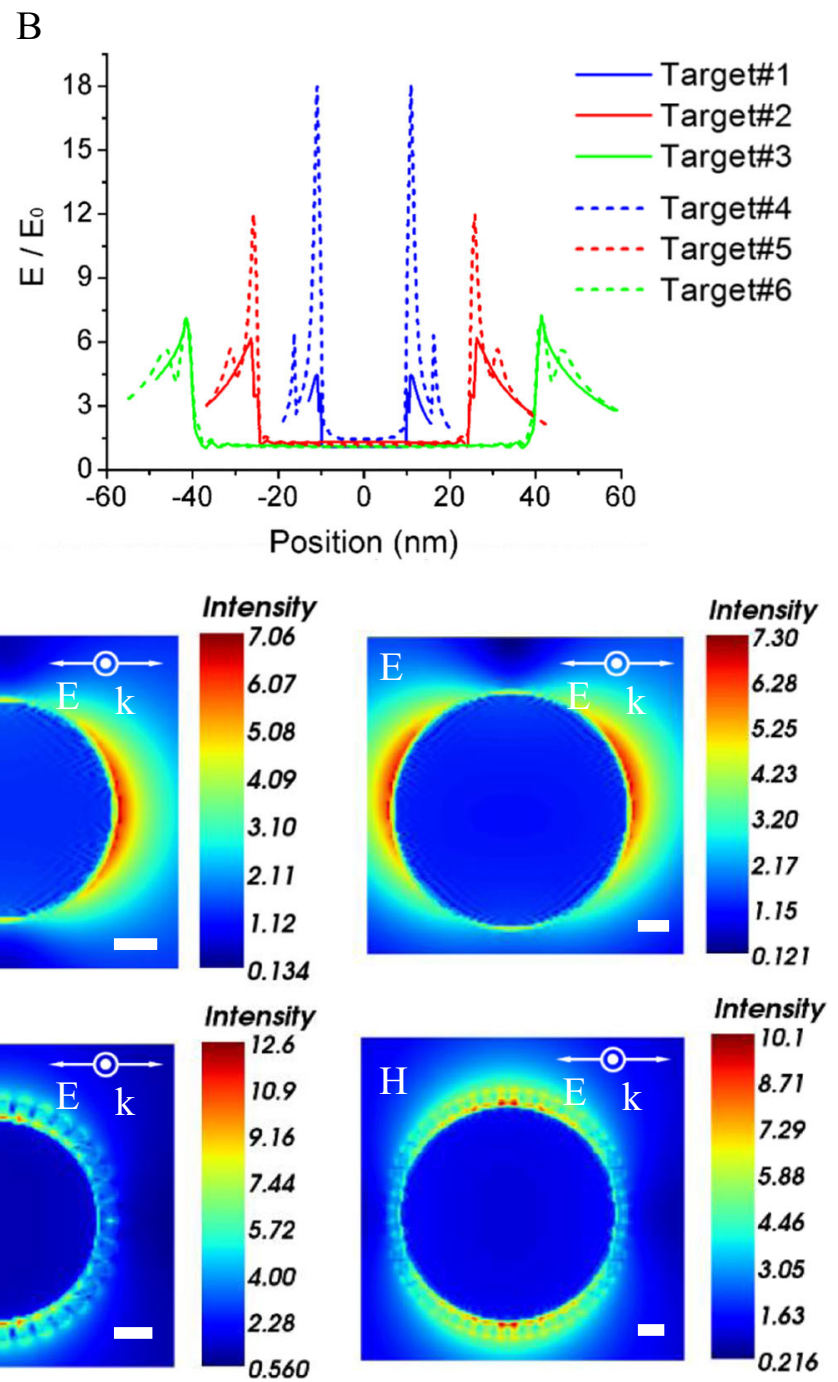

where $k=\frac{2 \pi}{\lambda}$ ( $\lambda$ is the wavelength), $R$ is the radius of the particles, and $F(m)$ is a function of the refractive index $(m)$. The absorption $\left(Q_{\mathrm{abs}}\right)$ can be written as:

$Q_{\mathrm{abs}}=4 \pi k R^{3} E(m)$

where $E(m)$ is also the function of the refractive index relying on a non-zero imaginary part. Thus, the ratio of scattering to absorbance is $\sim \frac{2}{3}(k R)^{3} \frac{F}{E}$, which is further equivalent to $(k R)^{3}$. Considering a wavelength of $\sim 500 \mathrm{~nm}$, for GNPs with $R$ equals to 10,25 , and $40 \mathrm{~nm}$, the value of $(k R)^{3}$ is 0.002 , 0.031 , and 0.127 , respectively. This result demonstrates a consistent trend with the ratio of scattering to absorbance in Fig. 5.

The extinction spectra for all the targets simulated here were further summarized in Fig. 6a. One can observe that large GNP and GNP-QD targets show significantly enhanced extinction as compared with the smaller ones. The trend from this simulation in Fig. 6a actually have a consistence with

distributions near/around the GNPs (c-e: targets \#1-\#3) and GNP-QD heterostructures (d-f: targets \#4 \#6). Note: Scale bar in $\mathbf{c}-\mathbf{h}$ : $10 \mathrm{~nm}$ 
these experimental results (Fig. 3). As observed, target \#3 and target \#6 (corresponding to GNP-120 and GNP-QD_3, respectively) showed a small difference in extinction efficiency. However, targets \#1 and \#3 (corresponding to GNP-45 and GNP-QD_1, respectively) showed the largest extinction efficiency difference among the three target sets. This is further confirmed by the normalized electric field imaging in Fig. 6b. At the same time, it is important to note that the extinction efficiency difference observed in the simulation results is not as large as that observed in experimental results. This is because of two reasons: (1) the simulation is conducted in an ideal environment for individual GNP or GNP-QD heterostructures, while in the experiment, the GNP or GNPQD heterostructures were exposed in ambient environment and further dispersed on a silicon substrate, which have significant reflection to the incident. This resulted in the deviation of experimental and simulation results. (2) In the simulation part, we assume that QDs are closely packed on the GNP surface. However, in the experiment, the absorbance was collected from a number of GNPs or GNP-QD heterostructures. In addition, the QDs were not tightly packed on the surface of GNPs. Since the GNP-45 sample had the highest surface coverage of quantum dots, it showed highest absorbance increase in experimental.

Normalized electric field distribution on or near the surface of the targets was further generated under the incident wavelength corresponding to the maximum of visible extinction peak of each target (Fig. 6c-h). For GNPs, the strongest electric filed is accumulated on the surface of the spherical targets, where the incident electromagnetic wave was parallel to the localized surface area vector. With respect to the GNP-QD heterostructures, the strongest electric field areas (normally called "hot spots") were mainly accumulated at the interface of GNP and quantum dots.

The variation of normalized electric field intensity $\left(|\mathrm{E}| /\left|\mathrm{E}_{0}\right|\right)$ along the central line of the target across the incident direction was shown in Fig. 6b. The symmetrical sharp peaks indicate the strongest electric field accumulated at the surface of GNPs or the interface of GNP-QD targets. One can also observe that GNPs show enhanced electric field near the surface with increased particle size. This trend is consistent with the extinction spectra showing in Fig. 6a. However, the varying trend of electric field intensity at the interface of GNP-QD heterostructures is reversed from the GNP targets. It was observed that large GNP and GNP-QD targets $\left(R_{\mathrm{GNP}}=40 \mathrm{~nm}\right)$ show equivalent electric field intensity, while small GNP-QD target $\left(R_{\mathrm{GNP}}=10 \mathrm{~nm}\right)$ shows significantly enhanced electric field at the "hot spot" as compared with the corresponding GNP. This again indicates strong light-matter interaction between small GNPs and quantum dots. Such simulation provides interesting fundamental knowledge for understanding and designing future plasmonic devices based on GNPs and GNP-QD heterostructures.

\section{Conclusions}

In summary, multilayer graphene shell encapsulated $\mathrm{Au}$ nanoparticle - quantum dot hybrids were fabricated using the electroless deposition, thermal annealing, CVD, and self-assembly chemistry. The fabrication process also demonstrates a unique combination of both, top-down and bottomup approaches. The thermal dewetting of Au films led to controlled patterning of $\mathrm{Au}$ nanoparticles, which further enabled patterned growth of GNPs in a CVD process. The presence of encapsulating multilayer graphene shell around $\mathrm{Au}$ nanoparticles enhanced the surface chemistry of the GNPs and allowed their linking with quantum dots in a simple carbodiimide functionalization approach. The highresolution electron microscopy showed that crystal structure of individual components present in the heterostructures or hybrids. UV-vis spectroscopy showed that presence of multilayer graphene shell did not suppress but modulated the absorbance peaks corresponding to encapsulated $\mathrm{Au}$ nanoparticles. GNPs could be successfully utilized as substrates for loading quantum dots without influencing the absorbance characteristics and their band gap energies. It was observed that fluorescence response for the quantum dots was quenched for the quantum dots loaded onto GNPs, and this was attributed to the electron-accepting characteristics of the latter. This fluorescence quenching was dependent on both, the size of GNPs and quantum dot loading on the GNPs. Meanwhile, the DDA simulation indicates that Au nanoparticles preserved their plasmonic properties after graphene encapsulation and quantum dot attachment. Strong light-matter interaction between GNP and quantum dots (especially for GNP with smaller size) was estimated.

Acknowledgments This work was supported by the National Science Foundation (Award \#: 0925445) and NSF-EPSCoR-RII award. The authors thank the University of Alabama's Office of sponsored programs and Research Grant Committee Award for additional support for this research. The authors also thank the Central Analytical Facility (CAF). They would also like to thank Dr. S. Kapoor for proofreading the manuscript.

Open Access This article is distributed under the terms of the Creative Commons Attribution 4.0 International License (http://creativecommons.org/licenses/by/4.0/), which permits unrestricted use, distribution, and reproduction in any medium, provided you give appropriate credit to the original author(s) and the source, provide a link to the Creative Commons license, and indicate if changes were made.

\section{References}

1. Liu H, Liang G, Abdel-Halim ES, Zhu JJ (2011) A sensitive and selective quantum dots-based FRET biosensor for the detection of 
cancer marker type IV collagenase. Anal Methods 3:1797-1801. doi:10.1039/C1AY05178D

2. Liu X, Yu M, Kim H, Mameli M, Stellacci F (2012) Determination of monolayer-protected gold nanoparticle ligand-shell morphology using NMR. Nat Commun 3:1182-1190. doi:10.1038/ ncomms 2155

3. Stobiecka M, Deeb J, Hepel M (2010) Ligand exchange effects in gold nanoparticle assembly induced by oxidative stress biomarkers: homocysteine and cysteine. Biophys Chem 146:98-107. doi:10. 1016/j.bpc.2009.11.001

4. Daniel MC, Astruc D (2004) Gold nanoparticles: assembly, supramolecular chemistry, quantum-size-related properties, and applications toward biology, catalysis, and nanotechnology. Chem Rev 104:293-346. doi:10.1021/cr030698+

5. Ribeiro T, Prazeres TJV, Farinha JPS (2013) Enhanced photoluminescence from micellar assemblies of cadmium sulfide quantum dots and Au nanoparticles. J Phys Chem C 117:31223133. doi:10.1021/jp311200r

6. Kulakovich O, Strekal N, Yaroshevich A, Maskevich S, Gaponenko S, Nabiev I, Artemyev M (2002) Enhanced luminescenece of CdSe quantum dots on Au colloids. Nano Lett 2:1449-1452. doi:10.1021/n1025819k

7. Peng X, Chen J, Misewich JA, Wong SS (2009) Carbon nanotubenanocrystal heterostructures. Chem Soc Rev 38:1076-1098. doi: 10.1039/B811424M

8. Haremza JM, Hahn MA, Krauss TD, Chen S, Calcines J (2002) Attachment of single CdSe nanocrystals to individual single-walled carbon nanotubes. Nano Lett 2:1253-1258. doi:10.1021/n1025799m

9. Mondal A, Jana NR (2014) Surfactant-free, stable noble metalgraphene nanocomposite as high performance electrocatalyst. ACS Catal 4:593-599. doi:10.1021/cs401032p

10. Wu J, Shi W, Chopra N (2014) Optical properties of Au/multilayergraphene/carbon nanotube hybrid materials. Carbon 68:708-717. doi:10.1016/j.carbon.2013.11.052

11. Kwon OS, Lee SH, Park SJ, An JH, Song HS, Kim T, Jang J (2013) Large-scale graphene micropattern nano-biohybrids: high-performance transducers for FET-type flexible fluidic HIV immunoassays. Adv Mater 25:4177-4185. doi:10.1002/adma.201301523

12. Li Y, Chopra N (2015) Progress in large-scale production of graphene. Part 1: chemical methods. JOM 67:34-43. doi:10.1007/ s11837-014-1236-0

13. Li Y, Chopra N (2015) Progress in large-scale production of graphene. Part 2: vapor methods. JOM 67:44-52. doi:10.1007/ s11837-014-1237-z

14. Wu J, Shi W, Chopra N (2012) Plasma oxidation kinetics of Au nanoparticles and their encapsulation in graphene shells by chemical vapor deposition growth. J Phys Chem C 116:12861-12874. doi:10.1021/jp301009f

15. Chopra N, Wu J, Summerville L (2013) Controlled assembly of graphene shells encapsulated Au nanoparticles and their integration with carbon nanotubes. Carbon 62:76-87. doi:10.1016/j.carbon. 2013.05.055

16. Chopra N, Bachas LG, Knecht MR (2009) Fabrication and biofunctionalization of carbon-encapsulated Au nanoparticles. Chem Mater 21:1176-1178. doi:10.1021/cm803349c

17. Sayed SY, Wang F, Malac M, Meldrum A, Egerton RF, Buriak JM (2009) Heteroepitaxial growth of Au nanostructures on silicon by galvanic displacement. ACS Nano 3(9):2809-2817. doi:10.1021/ nn900685a

18. Sayed SY, Wang F, Malac M, Li P, Wang D, Buriak J (2012) Preferential face deposition of Au nanoparticles on silicon nanowires by galvanic displacement. Crystallogr Eng Commun 14: 5230-5234. doi:10.1039/C2CE25254F

19. Sorgenfrei S, Chiu CY, Gonzalez RL Jr, Yu YJ, Kim P, Nuckolls C, Shepard KL (2011) Label-free single-molecule detection of DNA- hybridization kinetics with a carbon nanotube field-effect transistor. Nat Nanotechnol 6:126-132. doi:10.1038/nnano.2010.275

20. Li Y, Chopra N (2015) Graphene encapsulated gold nanoparticlequantum dot heterostructures and their electrochemical characterization. Appl Surf Sci 344:27-32. doi:10.1016/j.apsusc.2015.03. 072

21. Li Y, Chopra N (2015) Fabrication of nanoscale heterostructures comprised of graphene-encapsulated gold nanoparticles and semiconducting quantum dots for photocatalysis. Phys Chem Chem Phys. doi:10.1039/C5CP00928F, In press

22. Mane RS, Roh SJ, Joo OS, Lokhande CD, Han SH (2005) Improved performance of dense $\mathrm{TiO}_{2} / \mathrm{CdSe}$ coupled thin films by low temperature process. Electrochim Acta 50:2453-2459. doi:10. 1016/j.electacta.2004.10.075

23. Zhang $\mathrm{G}$, Wu H, Li G, Huang Q, Yang $\mathrm{C}$, Huang $\mathrm{F}$, Lin $\mathrm{J}$ (2013) New high Tc multiferroics $\mathrm{KBiFe}_{2} \mathrm{O}_{5}$ with narrow band gap and promising photovoltaic effect. Sci Rep 3:1265-1272. doi:10.1038/srep01265

24. Chen ML, Oh WC (2011) Synthesis and highly visible-induced photocatalytic activity of CNT-CdSe composite for methylene blue solution. Nanoscale Res Lett 6:1-8. doi:10.1186/1556-276X-6-398

25. Wei H, Su Y, Han Z, Li T, Ren X, Yang Z, Zhang Y (2013) $\mathrm{Zn}_{(\mathrm{x})} \mathrm{Cd}_{(1-\mathrm{x})} \mathrm{Se}$ nanomultipods with tunable band gaps: synthesis and first-principles calculations. Nanotechnology 24:235706235712. doi:10.1088/0957-4484/24/23/235706

26. Li Y, Shi W, Dykes J, Chopra N (2013) Growth of silicon nanowires-based heterostructures and their plasmonic modeling. MRS Proc 1547:103-108. doi:10.1557/opl.2013.542

27. Flatau PJ, Draine BT (1994) Discrete-dipole approximation for scattering calculations. J Opt Soc Am A 11:1491-1499. doi:10. 1364/JOSAA.11.001491

28. Draine BT, Flatau PJ (2013) User guide for the discrete dipole approximation code DDSCAT 7.3. arXiv preprint arXiv:1305.6497

29. Li Y, Dykes J, Chopra N (2013) Si nanowire-gold nanoparticles heterostructures for surface enhanced Raman spectroscopy. MRS Online Proc Libr 1551:mrss13-mrss1551. doi:10. 1557/opl.2013.900

30. Li Y, Chopra N (2014) Chemically modified and doped carbon nanotube-based nanocomposites with tunable thermal conductivity gradient. Carbon 77:675-687. doi:10.1016/j.carbon.2014.05.073

31. Li Y, Chopra N (2013) Nanoscale heterostructures comprised of silicon nanowires and gold nanoparticles encapsulated in graphitic shells for DNA immobilization. MRS Proc 1572:mrss13mrss 1572. doi:10.1557/opl.2013.908

32. Bittencourt C, Navio C, Nicolay A, Ruelle B, Godfroid T, Snyders R, Ewels CP (2011) Atomic oxygen functionalization of vertically aligned carbon nanotubes. J Phys Chem C 115:20412-20418. doi: 10.1021/jp2057699

33. Bruchez M, Moronne M, Gin P, Weiss S, Alivisatos AP (1998) Semiconductor nanocrystals as fluorescent biological labels. Science 281:2013-2016. doi:10.1126/science.281. 5385.2013

34. Bohren CF, Huffman DR (2008) Chapter 3. Absorption and scattering by an arbitrary particle. In: Absorption and scattering of light by small particles. John Wiley \& Sons, New York, p 57-81. doi: 10. 1002/9783527618156

35. Li Y, Chopra N (2014) Optical properties of nanostructured carbon and $\mathrm{Au}$ nanoparticle hybrids. MRS Proc 1700:mrss14-mrss 1700. doi:10.1557/opl.2014.575

36. Zhu S, Chen TP, Liu YC, Liu Y, Fung S (2012) A quantitative modeling of the contributions of localized surface plasmon resonance and interband transitions to absorbance of Au nanoparticles. J Nanoparticle Res 14:1-6. doi:10. 1007/s11051-012-0856-y 
37. Meng ZD, Zhu L, Ye S, Sun Q, Ullah K, Cho KY, Oh WC (2013) Fullerene modification $\mathrm{CdSe} / \mathrm{TiO} 2$ and modification of photocatalytic activity under visible light. Nanoscale Res Lett 8:1-10. doi:10. 1186/1556-276X-8-189

38. Samanta A, Zhou Y, Zou S, Yan H, Liu Y (2014) Fluorescence quenching of quantum dots by gold nanoparticles: a potential long range spectroscopic ruler. Nano Lett 14:5052-5057. doi:10.1021/ nl501709s

39. Zhang Z, Wang Z, Cao SW, Xue C (2013) Au/Pt nanoparticledecorated $\mathrm{TiO}_{2}$ nanofibers with plasmon-enhanced photocatalytic activities for solar-to-fuel conversion. J Phys Chem C 117:2593925947. doi:10.1021/jp409311x

40. Hou W, Cronin SB (2013) A review of surface plasmon resonanceenhanced photocatalysis. Adv Func Mater 23:1612-1619. doi:10. 1002/adfm. 201202148

41. Yamada K, Miyajima K, Mafuné F (2007) Thermionic emission of electrons from $\mathrm{Au}$ nanoparticles by nanosecond pulse-laser excitation of interband. J Phys Chem C 111:11246-11251. doi:10.1021/ jp0730747 